\title{
A novel perfusion culture system for screening mitochondrial toxicity in primary mouse hepatocytes
}

\author{
Chika Yamamoto', Akinori Takemura1, Sanae Ishii', Atsushi Doi², Isao Saito², \\ Hideki Yamada ${ }^{2}$, Yoko Sakai ${ }^{3}$, Tamihide Matsunaga ${ }^{3}$ and Kousei Ito' \\ 'Laboratory of Biopharmaceutics, Graduate School of Pharmaceutical Sciences, Chiba University, \\ 1-8-1 Inohana, Chuo-ku, Chiba 260-8675, Japan. \\ 2Shinko Chemical Co., Ltd, 2-4, Fujieminami, Kanazawa 920-0346, Japan \\ ${ }^{3}$ Department of Clinical Pharmacy, Graduate School and Faculty of Pharmaceutical Sciences, \\ Nagoya City University, 3-1 Tanabe-dori, Mizuho-ku, Nagoya 467-8603, Japan
}

(Received July 28, 2021; Accepted October 12, 2021)

\begin{abstract}
The liver microphysiological system (MPS) model is an in-vitro culture method that mimics physiological blood flow, which enhances basal cellular functions. However, the liver MPS model has not been tested in the preclinical stage because of its obscure utility. It can overcome the major problem of conventional systems - rapid loss of mitochondrial activity in cultured hepatocytes due to limited oxygen supply - by supplying oxygen to cultured hepatocytes using a perfusion device. In this study, we developed a new perfusion culture system that can detect mitochondrial toxicity. Primary mouse hepatocytes were cultured under perfusion condition for $48 \mathrm{hr}$. The hepatocytes showed increased oxygen consumption and reduced lactate release. These results indicated that the ATP-production pathway was switched from glycolysis to mitochondrial oxidative phosphorylation in the perfusion culture system. Furthermore, ATP levels were considerably reduced in the perfusion culture system after exposure to phenformin, a mitochondrial complex I inhibitor. To summarize, the perfusion culture system could improve the mitochondrial activity in primary mouse hepatocytes, and thus, has potential implications in the detection of mitochondrial toxicity.
\end{abstract}

Key words: Drug-induced liver injury, Mitochondrial toxicity, Perfusion culture, Primary mouse hepatocytes, Microphysiological system

\section{INTRODUCTION}

The microphysiological system (MPS) is a cell culture system having several connected compartments, similar to the body organs; culture medium circulates through the compartments to mimic the in-vivo conditions. Currently, many MPS models mimicking the major organs have been developed, which have improved specific functions of each organ compared with the conventional culture models (Kimura et al., 2018). Liver MPS models are also being developed (Baudy et al., 2020); however, their suitable utility is obscure. Therefore, liver MPS models need to be studied for their potential applications, including the evaluation of drug toxicity.

A large number of mitochondria are present in the liver because of its high demand for energy production. Mito- chondrial toxicity plays a central role in the pathogenesis of drug-induced liver injury (DILI) (Fromenty, 2020). More than half of the drugs in the black box warning by the U.S. Food and Drug Administration are responsible for mitochondrial toxicity (Dykens and Will, 2007). Therefore, mitochondrial toxicity assessment is considered important in the accurate prediction of DILI. However, the detection of mitochondrial toxicity using conventional culture systems is difficult as the cultured cells generate ATP via glycolysis rather than oxidative phosphorylation (Ikeyama et al., 2020a). Shifting from glycolysis to oxidative phosphorylation is difficult in conventional culture systems because of the lack of oxygen supply. A study has reported that the oxygen consumption rate of cultured hepatocytes is $40-90 \mathrm{pmol} / \mathrm{sec} / \mathrm{cm}^{2}$, and the oxygen supply rate through the liquid-vapor inter-

Correspondence: Kousei Ito (E-mail: itokousei@chiba-u.jp) 


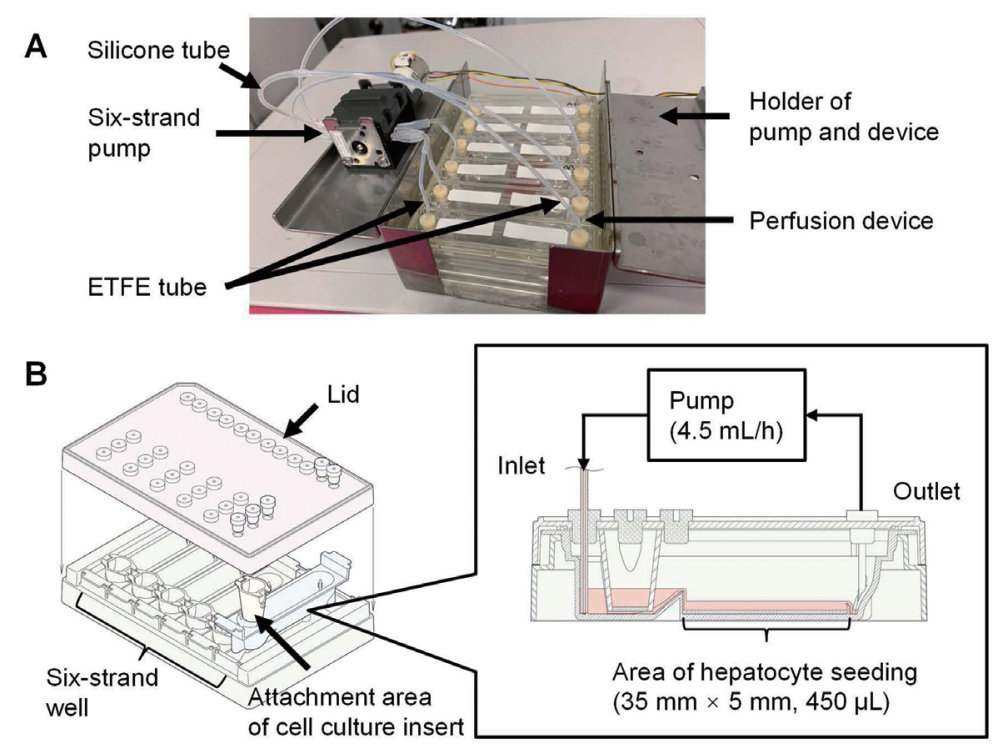

Fig. 1. Principle of the perfusion culture device. (A) A photograph image of the perfusion culture device. The perfusion culture device consisted of a device plate, six-strand pump, and tubes, and holder. (B) An illustration of the perfusion culture device. This device had six-strand well consisted of two compartments. One compartment near the inlet can be attached to a cell culture insert, whereas in the other compartment near the outlet, cells (hepatocytes in this case) can be seeded directly.

face is only $17 \mathrm{pmol} / \mathrm{sec} / \mathrm{cm}^{2}$ because of low oxygen solubility in culture medium (Sakai et al., 2012). We recently reported that the cells cultured with high oxygen concentration can avoid the energy shift from oxidative phosphorylation to glycolysis, and the sensitivity of mitochondrial toxicity in primary hepatocytes can be improved with drug treatment (Liu et al., 2016). Thus, the cells cultured with high oxygen supply can become a suitable model for detecting mitochondrial toxicity.

In the present study, we developed a perfusion culture system using a new device, as shown in Fig. 1. The perfusion device circulated the culture medium from the inlet to the outlet in six wells simultaneously. Previous report suggests that the perfusion culture enabled continuous oxygen supply to the cultured cells (Domansky et al., 2010), as oxygen consumed by cells was replenished by diffusive and convective flux from the liquid-vapor interface. To identify whether the perfusion culture could be a potential tool for assessing mitochondrial toxicity, we evaluated the mitochondrial function and sensitivity towards toxicants in primary mouse hepatocytes.

\section{MATERIALS AND METHODS}

\section{Fabrication of the perfusion device}

The perfusion culture device consisted of a device plate, pump, tubes, and holder (Fig. 1). The device plate consisted of six wells made of polystyrene, and was fabricated by injection molding. Each well consisted of an inlet, two compartments, and an outlet. One compartment near the inlet can be attached to an insert, whereas in the other compartment near the outlet, cells can be seeded directly. The dimensions of the latter compartment were approximately $35 \mathrm{~mm} \times 5 \mathrm{~mm}$. The following barriers were present in the device plate: i) one between the former compartment and the latter compartment, and ii) another between the latter compartment and the outlet. The height of two barriers was $5 \mathrm{~mm}$ and $2.2 \mathrm{~mm}$, respectively. The central region of each barrier was incised slightly to prevent pulsating flow. Plasma treatment and electron-beam sterilization were performed for hydrophilization and sterilization of the device plate, respectively. The device plate cover had several holes for connecting tubes and for supernatant collection. The perfusion system consisted of six strand-pumps (Takasago Electric, Inc., Aichi, Japan) connected to silicone tubes (outer diameter [O.D.]: $2 \mathrm{~mm}$, inner diameter [I.D.]: $1 \mathrm{~mm}$; AS ONE CORPORATION, Osaka, Japan) and ETFE tubes (O.D.: $1.6 \mathrm{~mm}$, I.D.: $0.5 \mathrm{~mm}$; Shimadzu corporation, Kyoto, Japan). This system could circulate the culture medium from the inlet to the outlet in six wells simultaneously. Two different tubes were connected in series for the following reasons. First, silicone tubes have high oxygen permeability to maintain oxygen concentration under perfusion culture conditions. Second, 
Perfusion culture device for mitochondrial toxicity screening

ETFE tubes have a lower dead volume than that of silicone tubes.

\section{Chemicals}

William's medium E (WME) and GlutaMAX were purchased from Thermo Fisher Scientific (Waltham, MA, USA). Fetal bovine serum (FBS) was purchased from Biosera (Nuaille, France). Insulin, transferrin, selenous acid (ITS) premix, collagen type I (Rat Tail), and Matrigel were purchased from Corning (Corning, NY, USA). Collagenase, insulin, and dexamethasone were purchased from FUJIFILM Wako Pure Chemical Industries (Osaka, Japan). Phenformin was purchased from Sigma-Aldrich (St. Louis, MO, USA). An antibiotic-antimycotic solution was purchased from Nacalai Tesque (Kyoto, Japan).

\section{Animals}

C57BL/6J male mice (Charles River Laboratories Japan Inc., Kanagawa, Japan), 7-10 weeks old, were used in this study. The animals were treated humanely according to the guidelines published by the National Institutes of Health (Bethesda, MD, USA). All animal experiments were approved by the Animal Care Committee of the Chiba University (Chiba, Japan).

\section{Isolation, purification, and seeding of hepatocytes}

Mouse hepatocytes were isolated by the two-step perfusion method, which has been reported previously (Ikeyama et al., 2020a). The hepatocytes were suspended in plating medium (WME supplemented with an antibiotic-antimycotic solution, $5 \% \mathrm{FBS}, 4 \mu \mathrm{g} / \mathrm{mL}$ insulin, $1 \mu \mathrm{M}$ dexamethasone, GlutaMAX, and $15 \mathrm{mM}$ HEPES, $\mathrm{pH}$ 7.4). The hepatocyte suspension was poured onto "device-plates" coated with collagen type I (Rat Tail) at a density of $1.00 \times$ $10^{5}$ cells $/ \mathrm{cm}^{2}$.

\section{Static/perfusion culture conditions}

A schematic of static/perfusion culture conditions is shown in Fig. 2. Approximately $4 \mathrm{hr}$ after cell plating, old medium was replaced with fresh medium. After $24 \mathrm{hr}$ of plating, $0.25 \mathrm{mg} / \mathrm{mL}$ Matrigel dissolved in $450 \mu \mathrm{L}$ of icecold culture medium (WME supplemented with antibiotic-antimycotic, ITS premix, $1 \mu \mathrm{M}$ dexamethasone, and GlutaMAX) for $24 \mathrm{hr}$ was overlaid on it. For the static culture condition, $450 \mu \mathrm{L}$ of culture medium was poured in each well. For the perfusion culture condition, $1280 \mu \mathrm{L}$ of culture medium was poured in each well and the flow rate was maintained $4.5 \mathrm{~mL} / \mathrm{hr}$, which was the upper limit that does not damage the pump unit. The medium was replaced with fresh medium daily for up to 4 days for both the cultures. The hepatocytes were incubated at $37^{\circ} \mathrm{C}$ in a humidified atmosphere with $95 \%$ air and $5 \% \mathrm{CO}_{2}$.

\section{Measurement of oxygen consumption rate}

On days 3 and 4, the oxygen consumption of mouse hepatocytes cultured in static or perfusion conditions was measured using an oxygen sensor probe (FireSting oxygen monitor, Pyroscience GmbH, Aachen, Germany). In case of perfusion culture, the oxygen consumption rate was measured immediately after perfusion was stopped. The medium was aspirated and replaced with fresh medium. The oxygen probe was fixed at $650 \mathrm{~nm}$ above the seeded cell. Oxygen tension was continuously monitored every $5 \mathrm{~min}$ for $20 \mathrm{~min}$. The oxygen concentration was defined as the percentage of the initial value (at time 0 ).

\section{Measurement of lactate production}

The medium was aspirated and replaced with fresh medium. The hepatocytes cultured under each culture condition were incubated at $37^{\circ} \mathrm{C}$ for $2 \mathrm{hr}$ under static conditions. After incubation, the medium was collected and lactate production was assessed. The lactate level in the medium was measured using Lactate Assay Kit-WST (DOJINDO Laboratories, Kumamoto, Japan).

\section{Assessment of drug exposure and ATP level}

Drug exposure was assessed using two methods. First, mouse hepatocytes cultured under each culture condition for 4 days were treated with phenformin $(0,150$, and $300 \mu \mathrm{M})$ under static conditions for $4 \mathrm{hr}$. Second, mouse hepatocytes cultured under each culture condition for 4 days were treated with phenformin for $4 \mathrm{hr}$ in static or perfusion condition, respectively, same as the culture condition. Considering the difference in medium volume (static, $450 \mu \mathrm{L}$; perfusion, $1280 \mu \mathrm{L}$ ), the cells were treated with $300 \mu \mathrm{M}$ (static) or $105 \mu \mathrm{M}$ (perfusion) phenformin to equalize the phenformin level. After drug exposure, the ATP level in cells was measured using the CellTiter-Glo luminescent cell viability assay kit (Promega, Madison, WI, USA). Luminescence was measured using a FilterMax F5 instrument (Molecular Devices, San Jose, CA, USA).

\section{Statistical analysis}

All data are presented as mean \pm standard deviation. The GraphPad Prism 7 software (GraphPad Software, La Jolla, CA, USA) was used to perform all statistical analyses. Data were analyzed using Student's t-tests or analysis of variance, followed by Dunnett's test to identify significant differences. A $p$-value of $<0.05$ was considered statistically significant. 


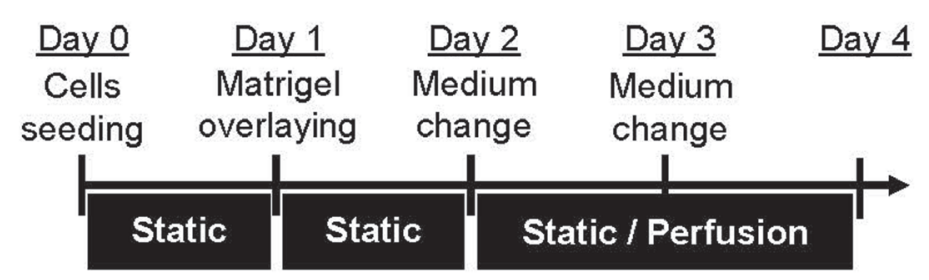

Fig. 2. Time course of the experimental procedure. On day 0, primary mouse hepatocytes were seeded onto a plate, and Matrigel was overlaid on it on day 1 . Culture medium was replaced with fresh medium every day. Perfusion culture was started from day 2 to day 4 , and the flow rate was maintained at $4.5 \mathrm{~mL} / \mathrm{hr}$.
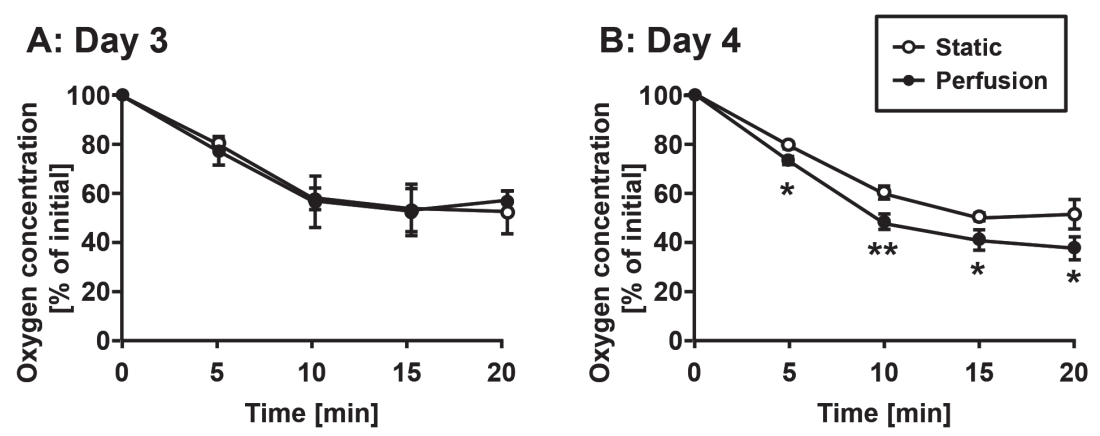

Fig. 3. Measurement of oxygen consumption rate in primary mouse hepatocytes cultured under each condition. Primary mouse hepatocytes were cultured as mentioned in Fig. 2. On day 3 (A) and day 4 (B), oxygen concentration was measured 20 min after changing the medium. Data were normalized to the initial oxygen concentration in the medium (100\%). Data are represented as means \pm standard deviation $(\mathrm{SD}) .(\mathrm{n}=3) .{ }^{*} p<0.05 .{ }^{*} p<0.01$ vs. static condition at the same time point.

\section{RESULTS}

To examine the effect of perfusion culture on mitochondrial function, we first evaluated the oxygen consumption rate, which represents mitochondrial activity. As shown in Fig. 3A, the oxygen consumption rate on day 3 showed no difference between the static and perfusion cultures. In contrast, the oxygen consumption rate in mouse hepatocytes cultured under perfusion condition increased on day 4, compared with static condition (Fig. 3B). Lactate, a major product of glycolysis under anaerobic conditions, is an indicator of glycolysis activity. As shown in Fig. 4, the lactate levels in mouse hepatocytes cultured under each condition did not differ on day 3 . On the other hand, the lactate level in mouse hepatocytes cultured under perfusion condition decreased on day 4 compared with that in those cultured under static culture conditions.

We next examined whether the sensitivity to mitochondrial toxicity was increased in mouse hepatocytes cultured under perfusion condition. We used phenformin, which is a mitochondrial respiratory chain complex I inhibitor (Dykens et al., 2008). Mouse hepatocytes were cultured as mentioned in Fig. 2, then treated with phenformin under static condition for $4 \mathrm{hr}$. ATP level did not change in mouse hepatocytes cultured under static conditions with $150 \mu \mathrm{M}$ phenformin. On the other hand, ATP levels in mouse hepatocytes cultured under perfusion condition were significantly reduced after treatment with $300 \mu \mathrm{M}$ phenformin (Fig. 5A). Next, we evaluated ATP level after phenformin treatment for $4 \mathrm{hr}$ under static or perfusion conditions. As shown in Fig. 5B, ATP levels in mouse hepatocytes cultured under perfusion condition markedly decreased compared with those in mouse hepatocytes cultured under static condition. In addition, ATP levels in control group ( $0 \mu \mathrm{M}$ phenformin) had no significant difference in each culture conditions (Supplement Fig. 2A and B).

\section{DISCUSSION}

Liver MPS models can efficiently improve cell functions compared with the conventional culture models (Dash et al., 2013). However, liver MPS models have 
Perfusion culture device for mitochondrial toxicity screening

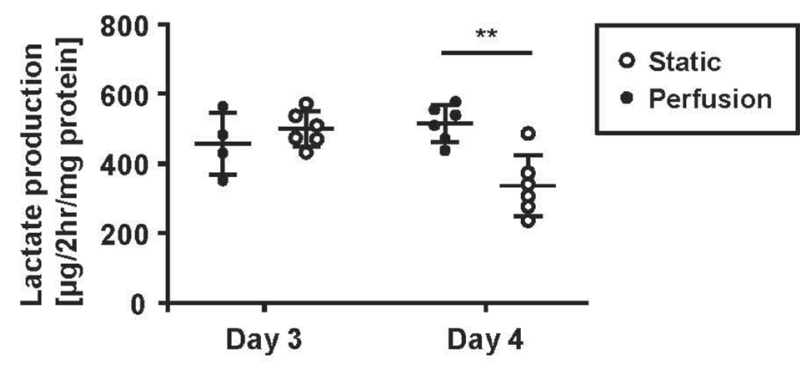

Fig. 4. Measurement of lactate level in primary mouse hepatocytes cultured under each condition. Primary mouse hepatocytes were cultured as mentioned in Fig. 2. On day 3 (A) and day 4 (B), the cells cultured under each condition were incubated for $2 \mathrm{hr}$ under static condition, and lactate level in the supernatant was measured. Data are represented as means $\pm \mathrm{SD}$. $(\mathrm{n}=4-6) .{ }^{*} p<0.01$.

not been tested in the preclinical stage. The present study showed the potential use of a newly developed MPS using a perfusion culture system for assessing mitochondrial toxicity. On the basis of oxygen consumption (Fig. 3B) and lactate production (Fig. 4), the present study suggested that the perfusion culture system can more efficiently improve mitochondrial activity than the static culture system. Furthermore, the perfusion culture system increased the sensitivity of mouse hepatocytes to phenformininduced mitochondrial toxicity (Fig. 5A and 5B).

Previously, we reported that primary mouse hepatocytes cultured under $40 \%$ oxygen concentration activate mitochondrial function (Ikeyama et al., 2020a). The study did not measure oxygen consumption, but it is possible that oxygen consumption was also doubled because the oxygen concentration conditions were twice normal. In the present study, it is theoretically considered that the oxygen consumption rate of primary mouse hepatocytes under perfusion condition increased about twice as much as that under static condition (Supplement Fig. 1A, B and supplement Table 1). Because of abundant oxygen supply, ATP production pathway was switched from glycolysis to mitochondrial oxidative phosphorylation, and thus the ATP level in mouse hepatocytes cultured under perfusion condition was reduced after phenformin treatment on day 4 (Fig. 5A and 5B). The perfusion culture system affected cell function and viability not only through abundant oxygen supply but also through shear stress. Optimal shear stress affects cellular functions and increases sensitivity to drug toxicity (Rashidi et al., 2016). Shear stress applied in perfusion devices might be an important factor for mimicking physiological conditions. More investigation is needed to determine the relationship between shear stress in perfusion culture and drug toxicity.

To maintain a high oxygen concentration, we circulated the culture medium through silicone tubes. However, the use of silicone tubes leads to the adsorption of low molecular compounds. Generally, the adsorption of low molecular compounds is related to their lipophilic property (Wang et al., 2012). Our preliminary experiment showed that phenformin ( $\log \mathrm{P}$ value: -0.83$)$ was hardly adsorbed into the silicone tubes, whereas the drugs with high lipophilicity (rotenone, ketoconazole, and imipramine [ $\log$ P value: $4.1,4.35$, and 4.8 , respectively]) were easily adsorbed into silicone tubes (data not shown). For the industrial application of perfusion devices, the problem of drug adsorption into tubes need to be addressed.

MPS is expected to replace animal experiments in drug development process (Beckwitt et al., 2018). Cryopreserved human hepatocytes $(\mathrm{CHH})$ are the gold stand-
A: Exposure in static

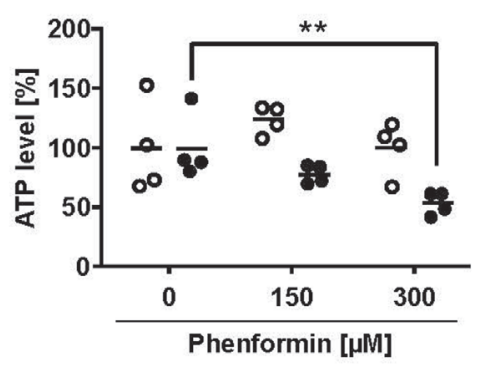

\section{B: Exposure in perfusion}

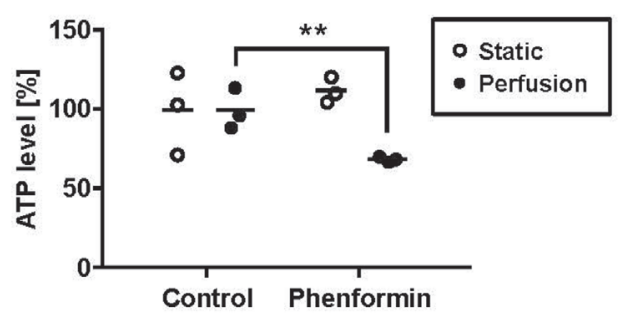

Fig. 5. The effect of perfusion culture on the sensitivity of phenformin-induced mitochondrial toxicity. Primary mouse hepatocytes were cultured as mentioned in Fig. 2. (A) The cells were exposed to phenformin for $4 \mathrm{hr}$ in static condition and the ATP level was measured. (B) The cells were exposed to phenformin for $4 \mathrm{hr}$ in static or perfusion condition and the ATP level was measured. Data are represented as means $\pm \mathrm{SD}$. $(\mathrm{n}=3-4) .{ }^{* *} p<0.01$. 
ard for the evaluation of liver injury, and are widely used in liver MPS models (Beckwitt et al., 2018). However, mitochondrial toxicity assays using $\mathrm{CHH}$ have a drawback that the cryopreservation process damages mitochondria (Ikeyama et al., 2020b). Therefore, the mitochondria present in $\mathrm{CHH}$ cannot be activated in perfusion culture. Thus, the use of primary mouse hepatocytes is appropriate. The mitochondrial sensitivity in $\mathrm{CHH}$ cultured in 3D spheroid culture toward toxicants was higher than that in the 2D sandwich culture (Bell et al., 2018). Taken together, if we construct a $\mathrm{CHH}$ mitochondrial toxicity assay based on the present device, we need to combine other methods such as 3D spheroid configuration with the perfusion culture.

To summarize, the mitochondrial activity in primary mouse hepatocytes cultured in perfusion culture was improved. Moreover, the sensitivity to mitochondrial complex I inhibitor, phenformin in mouse hepatocytes cultured in perfusion culture was increased. However, to prove potential of predicting mitochondrial toxicity using perfusion culture, further examination is required to evaluate other mitochondrial toxicants. Understanding MPS characteristic and testing the suitability of the model for industrial applications is important.

\section{ACKNOWLEDGMENTS}

This research was supported by AMED under Grant Number JP21be0304203. We would like to thank Editage (www.editage.com) for English language editing.

Conflict of interest---- The authors declare that there is no conflict of interest.

\section{REFERENCES}

Baudy, A.R., Otieno, M.A., Hewitt, P., Gan, J., Roth, A., Keller, D., Sura, R., Van Vleet, T.R. and Proctor, W.R. (2020): Liver microphysiological systems development guidelines for safety risk assessment in the pharmaceutical industry. Lab Chip, 20, 215-225.

Beckwitt, C.H., Clark, A.M., Wheeler, S., Taylor, D.L., Stolz, D.B., Griffith, L. and Wells, A. (2018): Liver 'organ on a chip'. Exp. Cell Res., 363, 15-25.
Bell, C.C., Dankers, A.C., Lauschke, V.M., Sison-Young, R., Jenkins, R., Rowe, C., Goldring, C.E., Park, K., Regan, S.L., Walker, T., Schofield, C., Baze, A., Foster, A.J., Williams, D.P., van de Ven, A.W., Jacobs, F., Houdt, J.V., Lähteenmäki, T., Snoeys, J., Juhila, S., Richert, L. and Ingelman-Sundberg, M. (2018): Comparison of hepatic 2D sandwich cultures and 3D spheroids for long-term toxicity applications: A multicenter study. Toxicol. Sci., 162, 655-666.

Dash, A., Simmers, M.B., Deering, T.G., Berry, D.J., Feaver, R.E., Hastings, N.E., Pruett, T.L., LeCluyse, E.L., Blackman, B.R. and Wamhoff, B.R. (2013): Hemodynamic flow improves rat hepatocyte morphology, function, and metabolic activity in vitro. Am. J. Physiol. Cell Physiol., 304, C1053-C1063.

Domansky, K., Inman, W., Serdy, J., Dash, A., Lim, M.H. and Griffith, L.G. (2010): Perfused multiwell plate for 3D liver tissue engineering. Lab Chip, 10, 51-58.

Dykens, J.A., Jamieson, J., Marroquin, L., Nadanaciva, S., Billis, P.A. and Will, Y. (2008): Biguanide-induced mitochondrial dysfunction yields increased lactate production and cytotoxicity of aerobically-poised HepG2 cells and human hepatocytes in vitro. Toxicol. Appl. Pharmacol., 233, 203-210.

Dykens, J.A. and Will, Y. (2007): The significance of mitochondrial toxicity testing in drug development. Drug Discov. Today, 12, 777-785.

Fromenty, B. (2020): Alteration of mitochondrial DNA homeostasis in drug-induced liver injury. Food Chem. Toxicol., 135, 110916.

Ikeyama, Y., Sato, T., Takemura, A., Sekine, S. and Ito, K. (2020a): Hypoxia/reoxygenation exacerbates drug-induced cytotoxicity by opening mitochondrial permeability transition pore: possible application for toxicity screening. Toxicol. In Vitro, 67, 104889.

Ikeyama, Y., Sato, T., Takemura, A., Sekine, S. and Ito, K. (2020b): Successful energy shift from glycolysis to mitochondrial oxidative phosphorylation in freshly isolated hepatocytes from humanized mice liver. Toxicol. In Vitro, 65, 104785.

Kimura, H., Sakai, Y. and Fujii, T. (2018): Organ/body-on-a-chip based on microfluidic technology for drug discovery. Drug Metab. Pharmacokinet., 33, 43-48.

Liu, C., Sekine, S. and Ito, K. (2016): Assessment of mitochondrial dysfunction-related, drug-induced hepatotoxicity in primary rat hepatocytes. Toxicol. Appl. Pharmacol., 302, 23-30.

Rashidi, H., Alhaque, S., Szkolnicka, D., Flint, O. and Hay, D.C. (2016): Fluid shear stress modulation of hepatocyte-like cell function. Arch. Toxicol., 90, 1757-1761.

Sakai, Y., Nishikawa, M., Evenou, F., Hamon, M., Huang, H., Montagne, K.P., Kojima, N., Fujii, T. and Niino, T. (2012): Engineering of implantable liver tissues. Methods Mol. Biol., 826, 189-216.

Wang, J.D., Douville, N.J., Takayama, S. and ElSayed, M. (2012): Quantitative analysis of molecular absorption into PDMS microfluidic channels. Ann. Biomed. Eng., 40, 1862-1873. 